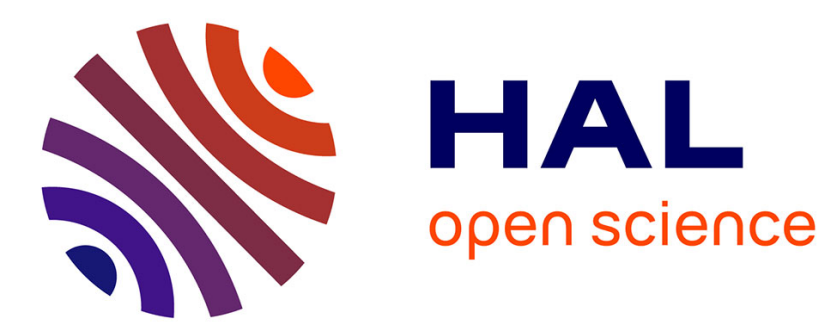

\title{
Single-Molecule Force Spectroscopy: Experiments, Analysis, and Simulations
}

\author{
Fidan Sumbul, Felix Rico
}

\section{To cite this version:}

Fidan Sumbul, Felix Rico. Single-Molecule Force Spectroscopy: Experiments, Analysis, and Simulations. Atomic Force Microscopy, Methods in Molecular Biology, pp.163-189, 2019. hal-02272098

\section{HAL Id: hal-02272098 \\ https://hal-amu.archives-ouvertes.fr/hal-02272098}

Submitted on 27 Aug 2019

HAL is a multi-disciplinary open access archive for the deposit and dissemination of scientific research documents, whether they are published or not. The documents may come from teaching and research institutions in France or abroad, or from public or private research centers.
L'archive ouverte pluridisciplinaire HAL, est destinée au dépôt et à la diffusion de documents scientifiques de niveau recherche, publiés ou non, émanant des établissements d'enseignement et de recherche français ou étrangers, des laboratoires publics ou privés. 
SINGLE-MOLECULE FORCE SPECTROSCOPY: EXPERIMENTS, ANALYSIS

\section{AND SIMULATIONS}

Fidan Sumbul ${ }^{1}$, Felix Rico $^{1 *}$

${ }^{1}$ U1006 INSERM, Aix-Marseille Université, Parc Scientifique et Technologique de Luminy, 163 avenue de Luminy, 13009 Marseille, France.

\section{Correspondence:}

felix.rico@inserm.fr

Running Head: Experimental and Computational Force Spectroscopy 


\section{ABSTRACT}

The mechanical properties of cells and of subcellular components are important to obtain a mechanistic molecular understanding of biological processes. The quantification of mechanical resistance of cells and biomolecules using biophysical methods matured thanks to the development of nanotechnologies such as optical and magnetic tweezers, the biomembrane force probe and atomic force microscopy (AFM). The quantitative nature of force spectroscopy measurements has converted AFM into a valuable tool in biophysics. Force spectroscopy allows the determination of the forces required to unfold protein domains and to disrupt individual receptor/ligand bonds. Molecular simulation as a computational microscope allows investigation of similar biological processes with an atomistic detail. In this chapter, we first provide a step-by-step protocol of force spectroscopy including sample preparation, measurement and analysis of force spectroscopy using AFM and its interpretation in terms of available theories. Next, we present the background for molecular dynamics (MD) simulations focusing on steered molecular dynamics (SMD) and the importance of bridging of computational tools with experimental techniques

Keywords: Dynamic Force Spectroscopy, Atomic Force Microscopy, Steered Molecular Dynamics Simulations, Receptor-Ligand interactions

\section{INTRODUCTION}

The mechanics of biological systems is crucial for their function [1]. Individual proteins require flexibility of secondary structures to allow microsecond conformational changes [2]. In the living cell, shape maintenance and structural stability is assured by the mechanics of the cytoskeleton, adhesion complexes and the plasma membrane [3]. Thus, the study of the mechanics of proteins, protein complexes and the membrane is important to understand biological processes. 
Interaction between two biomolecules such as proteins, DNA, RNA or drug molecules is the key phenomena in many biological processes. Their function depends on recognizing and binding to each other in the cell environment. Until recently, protein-protein interactions were characterized through binding affinities and/or rate constants determined by biochemical methods. Since these experimental methods are bulk measurements, they can only give averaged estimates of the desired properties. However, proteins exist in an ensemble of conformations where their populations change with varying conditions, Thus, the characterization of the interaction dynamics that expose various intermediate states or alternative reaction pathways is critical [4]. Single molecule approaches allow characterization of individual interacting molecules in real time and close to physiological conditions. With these attributes, they have become essential tools for understanding of association/disassociation processes of biomolecular complexes together with intermediate states and the underlying energy landscape [5-9].

The quantification of forces holding protein complexes together using biophysical methods has evolved with the development of nanotechnologies such as optical and magnetic tweezers and the biomembrane force probe $[10,11]$. One of the most versatile nanotechniques is atomic force microscopy (AFM), which enables topographical imaging and force measurements at the nanometre scale with piconewton force resolution. Importantly, AFM works under liquid conditions allowing characterization of biological samples. The flexible cantilever system is sensitive to picometer deflection changes and has associated a spring constant that can be as low as $\sim 6 \mathrm{pN} / \mathrm{nm}$ and, using micrometer size high-speed AFM cantilevers, the reachable time resolution is $<1$ microsecond $[12,13]$. Thus, it is an excellent force sensor that allows manipulation of the sample and force application. In force spectroscopy mode, the cantilever is moved in the vertical direction (z) to apply and measure mechanical forces by pulling or poking the sample. The quantitative nature of force spectroscopy measurements has converted AFM 
into a valuable tool in biophysics. Force spectroscopy allowed determining the forces required to unfold protein domains and to disrupt individual receptor/ligand bonds as well as probing the mechanical properties of normal and cancerous cells [14-24].

\subsection{Dynamic Force Spectroscopy (DFS) using AFM.}

\subsubsection{Principles of AFM Force Spectroscopy}

AFM was developed as an imaging tool where the topographic images of the sample are obtained by scanning the surface in the $\mathrm{x}$, y plane with a flexible cantilever [7]. The core components of an AFM device are: a flexible cantilever, featuring a tip at its free end, a piezoelectric translator that moves the sample stage or the cantilever depending on the design, and an optical deflection system composed of a laser, a segmented photodiode (PD) and a signal processing unit, which records the changes in cantilever deflection (Figure 1).

One of the applications of AFM is force spectroscopy, which allows us to measure the interaction between two surfaces, even at the single molecule level. In a typical AFM-based force spectroscopy setup for receptor-ligand interactions, a flexible cantilever functionalized with a molecule, is positioned close to the sample surface functionalized with the other molecule of interest. The AFM tip, with an average apex diameter of $10-50 \mathrm{~nm}$ diameter, narrows the interacting surfaces to the limits of single molecule interactions [15].

The position of the cantilever and the corresponding photodetector signal recorded during a typical force spectroscopy experiment is schematically shown in Figure 1.b. In the recorded signal, $y$-axis represents the photodetector signal proportional to the bending of the cantilever, converted to force by knowing the spring constant of the cantilever. The $\mathrm{x}$-axis in Fig 1 represents the time, although most works report the distance between the base of the piezo and the stationary surface, often termed extension. The typical force experiment begins with positioning of the functionalized cantilever a few hundreds of nanometres above the sample 
surface as in part I in Figure 1.b. The cantilever is then moved towards the sample surface with the help of piezo elements and makes contact with the sample surface (part II). The deflection signal is at a constant value (force) corresponding to "zero force" until the tip contact the surface. After the tip touches the sample surface, the cantilever is further pushed towards the sample surface until a pre-set deflection/force is reached to ensure the formation of the bonds between the molecules. This extra pushing causes the cantilever to bend upward leading to a positive change of the deflection/force signal (part III). After the peak force is reached, the cantilever starts to move back to its initial position. In the course of backward movement of the cantilever, the adhesive contacts formed while the tip is in contact with the sample surface are revealed causing a negative (See Note 1) deflection of the cantilever (part IV). If specific interaction is formed between the molecule functionalized on the tip and the molecule attached on the surface, the cantilever bends further downward (part V). In this part, further retraction movement of the cantilever from the contact point results in a gradual increase of the tension applied to the intermolecular bond(s) until the bond(s) rupture. This will cause a sharp change in deflection/force signal, and the cantilever returns to its original constant baseline (part VI). The result is the so-called force-extension/time curve or just force curve.

[Place Figure 1 here].

Dynamic force spectroscopy experiments consist of following this force curve acquisition at different retraction velocities. The typical range of velocities during dynamic force spectroscopy measurements accessible via conventional AFM extends from a few $\mathrm{nm} / \mathrm{s}$ to a few $\mu \mathrm{m} / \mathrm{s}$. The position of the reflected laser on the segmented photodiode is collected with a typical sampling rate of tens of kSamples/s $[10,25]$. The spring constant of commercial AFM cantilevers for single biomolecule measurements ranges between 6-100 pN/nm[4], following Hooke's law, resulting in forces range from $10 \mathrm{pN}$ to $1 \mu \mathrm{N}$ (the lower force being limited by thermal noise, $F_{t h}$, which depends on the viscous drag coefficient of the cantilever $(b)$, and not 
the spring constant, and the acquisition bandwidth (BW) as $5 F_{t h}=\sqrt{4 b k_{B} T B W}$ ) where $k_{B}$ is Boltzmann constant and $T$ is the absolute temperature.

\subsubsection{Surface and tip functionalization}

In dynamic force spectroscopy (DFS) experiments, one of the molecules (protein or ligand) is immobilized on the surface of a tip mounted at the end of the cantilever while the other is attached to the surface. Proper functionalization of the molecules to tip and surface is thus crucial for the success of this type of measurement. This is necessary to guarantee that the measured forces are specific to the studied intermolecular binding and that the molecules do not detach from tip or substrate. The most commonly used techniques to functionalize the molecules to the cantilevers are physisorption [15] and chemisorption [26]. In order to provide greater mobility and allow rearrangement of the molecule functionalized on the tip in order to access molecules on the surface, a linker molecule (usually a polyethylene glycol (PEG) linker) can be used to link the surface and the molecule of interest. Linkers of known length provide a signature of the specificity and valency of the measured force event $[27,28]$.

Similar immobilization techniques can be also used to functionalize the corresponding binding partners to a suitable flat surface. Mica and glass are common substrates used for functionalization of proteins to the surface because their silica-like structures allow the researcher to use similar surface chemistry as that used for the cantilevers.

Although both tip and surface functionalization protocols are well established, one should be aware of that strength of functionalization depends on the bioconjugation method used. For example, if a biotin-streptavidin interaction is going to be used in a multi-functionalization protocol of the relevant molecule to the AFM tip, one should keep in mind the range of biotinstreptavidin interaction forces (i.e. 20-400 pN). Thus, the quality of functionalization of both AFM tips and surfaces should be validated before performing actual force measurements. There 
are a number of alternative methods to bioconjugate molecules to surfaces. The use of specific tags that allow the formation of covalent bonds are of particular interest, as they provide both a strong linkage and a controlled orientation of the molecule. For a review on recent advances on this topic, see reference [29].

\subsubsection{Calibration of AFM cantilevers}

The change in the position of the deflected laser beam on the photodiode reflects the bending or deflection of the AFM cantilever due to the interaction forces between the tip and the sample. In order to convert the photodiode signal into units of force, the spring constant of the cantilever $(k)$ and the deflection sensitivity $(s)$ of the detector must be calibrated before any measurement [4]. The available techniques used in AFM to calibrate the cantilevers can be classified into two classes: 1) contact based and 2) contact-free methods.

\subsubsection{Contact Based Methods}

This is the most common method used in AFM. It involves determination of the photodiode sensitivity by acquiring a force-distance curve on a stiff surface (Fig. 2a) and then determination of the cantilever spring constant via thermal fluctuations of the cantilever (Fig. 2a), both in liquid.

In a force-distance curve acquired on a hard substrate, the measured deflection (in Volts) of the contact region is equal to the piezo movement (in $\mathrm{nm}$ ) [30]. The slope of the force-distance curve is the deflection sensitivity $(s)$ and its inverse, the optical lever sensitivity (often referred to as invOLS, Figure 2.a) [31-34]. To reliably determine the invOLS in the contact region, the forces applied should be high enough to minimize any effect from long-range repulsion forces between the tip and the surface, possible indentation of debris or contamination of the surfaces and thus assure that the piezo movement directly translates into deflection. Since relatively high 
contact forces are required for this sensitivity measurement, there is a risk to damage the cantilever tip or, importantly, the coating.

Different techniques are used for the cantilever spring constant calibration. The most popular is likely the Hutter and Bechhoefer method or thermal method [35]. After the deflection sensitivity is determined, recording the thermal fluctuations of the cantilever away from the surface and calculating the thermal spectrum allows estimation of the spring constant of the cantilever $(k)$. In this method, the cantilever is considered as a simple harmonic oscillator and the spring constant is derived from the power spectrum of thermal fluctuations of the cantilever, invoking the equipartition theorem, i.e. equating the thermal energy calculated from the absolute temperature $\left(k_{B} T\right)$ to the elastic energy from the oscillation of the cantilever [35]. The first resonance peak which has the largest amplitude and the best signal-to-noise ratio is usually used for the calibration of the spring constant. Two main corrections are required when using the optical lever method to detect the cantilever deflection, as most AFM systems use, and the thermal method is applied. First, correction for the different oscillation modes of the cantilever (first mode contributing 0.971). And second, correction for the difference in the bending of the cantilever between supported (force curve) or non-supported (thermal fluctuations) configurations [31, 36-39]. Since the cantilever deflection during force curves is in contact (supported end), the bending profile is different from that during the thermal fluctuations measurement (non-supported or free end). This requires a correction of the cantilever deflection by 1.09 , thus, squared in the mean squared deflection $\left(<x^{2}>\right)$. Therefore, including the combined corrections: $k=0.817 \frac{k_{B} T}{<x^{2}>}$. For a detailed description see Ref. 34 . 


\subsubsection{Contact-free Methods}

Contact free methods rely on prior knowledge of the spring constant of the cantilever and the use of the thermal fluctuations in liquid (Fig. 2b) to determine the photodiode sensitivity, somehow inversely to the last step of the contact method.

There are different methods to calibrate the spring constant of cantilevers. For example, detecting the thermal motion of the cantilever by using a laser Doppler vibrometer that directly measure the deflection in length units of the cantilever. However, this is difficult to implement in practice and requires expensive instrumentation.

Another method, known as the Sader method [40] is easily implemented using common AFM software. It uses the plan view dimensions of the cantilever (measured by optical or scanning electron microscopy), its resonance frequency and quality factor $(Q)$ in air (the method is valid only for high $Q$, See Note 2), and the physical properties of the environment used (the density and viscosity). The resonance frequency and quality factor $(Q)$ of the cantilevers are determined from the power spectral density (PSD) of the thermal vibration response of a cantilever (See Figure 2.b). This method was originally developed for rectangular shaped cantilevers and then extended to arbitrary shaped cantilevers $[41,42]$. Since the calibration of the spring constant of the cantilever using the Sader method is independent of the determination of detector sensitivity (invOLS), it is a convenient method, as it prevents damaging the cantilever tip or the coating.

Recently, Sader and co-workers initiated a web-based platform for spring constant calibration, the Global Calibration Initiative (GCI). Using this portal, any AFM user can upload the calibration parameters (spring constant, resonance frequency and Q-factor) of their own cantilevers, establishing a global database [43]. The assessment of the uploaded data from the individual users facilitates calculation of a universal coefficient, called $A$-coefficient, for a specific cantilever geometry which completes the functional relation between the spring 
constant $(k)$, the resonance frequency $\left(f_{R}\right)$ and the quality factor $(Q)$ measured in air [43]. The portal also allows the determination of the spring constant using the universal $A$-coefficient. The spring constant determination via GCI becomes more and more accurate as users upload their own calibrations to the database.

Other corrections to be applied to the force are due to the inherent tilt formed by the cantilever and the sample surface (often of $\sim 10^{\circ}$ ) and the torque induced by the tip height. For a detailed analysis see [44] and the following comment to it [45]. For conventional cantilevers, with a high ratio of tip height versus cantilever length, these corrections are $<5 \%$, but can be larger for the shorter high-speed AFM cantilevers [46].

[Place Figure 2 here].

\subsubsection{Interpretation of the Results}

Although other models allow determining more parameters of the energy landscape describing the unbinding processes, the simplest Bell-Evans model should be used unless the force spectrum reveals a nonlinear profile [47-51]. The Bell-Evans model is based on transition-state theory originally formulated by Kramers [52, 53], where two relatively stable states (bound and unbound states) of the molecules are separated by a free energy barrier (the transition state) at a distance $x_{\beta}$ from the bound state. The energy difference between bound and the transition state is $\Delta G^{0}$, defined as the activation energy. The dissociation rate $k_{o f f}$ of this complex can be expressed as

$$
k_{o f f}=\alpha \frac{k_{B} T}{h} \exp \left(\frac{-\Delta G^{0}}{k_{B} T}\right)
$$


where $\alpha$ is a prefactor identifying the potential energy well, $k_{B}$ is Boltzmann's constant, $T$ is the absolute temperature, $h$ is Planck's constant, and $\Delta G^{0}$ is the activation energy [4]. The dissociation rate in the presence of a constant pulling force $f$ becomes

$$
k_{o f f}(f)=\alpha \frac{k_{B} T}{h} \exp \left(\frac{-\left(\Delta G^{0}-f x_{\beta}\right)}{k_{B} T}\right)=k_{o f f} \exp \left(\frac{f x_{\beta}}{k_{B} T}\right)
$$

As can be seen from Equation 1.2, the dissociation rate of the complex exponentially increases with force. This expression was initially derived by Bell in the context of biological bonds, and later extended by Evans and Ritchie for a linearly increasing force (loading rate). Accordingly, the probability density function for the forced unbinding of a complex under constant loading rate $\left(r_{f}\right)$ is,

$$
P(f)=k_{o f f} \exp \left(\frac{f x_{\beta}}{k_{B} T}\right) \exp \left\{\frac{k_{o f f} k_{B} T}{r_{f} x_{\beta}}\left[1-\exp \left(\frac{f x_{\beta}}{k_{B} T}\right)\right]\right\}
$$

Then, the most probable rupture force $f^{*}$ (i.e., the maximum of the probability distribution function $\partial P(f) / \partial f=0$ ) is obtained from Equation 1.3 as

$$
f^{*}=\frac{k_{B} T}{x_{\beta}} \ln \left(\frac{x_{\beta}}{k_{o f f} k_{B} T}\right)+\frac{k_{B} T}{x_{\beta}} \ln \left(r_{f}\right)
$$

Equation 1.4 reveals the linear dependence of the most probable rupture force $f^{*}$ (see Note 3) with the logarithm of the loading rate $r_{f}$. Therefore, the kinetic parameters of the dissociation process, are obtained from the slope and intercept of the best linear fit of Equation 1.2 to the dynamic force spectrum: $f^{*}$ versus $\ln \left(r_{f}\right)$ [4]. Alternatively, the density function Equation 1.3 can be directly used to globally fit the rupture force histograms to extract the landscape 
parameters. However, due to uncertainties in the determination of the force baseline, thermal fluctuations on the cantilever and other sources, common histograms appear wider than predicted by Equation 1.3. Moreover, if the distance to the transition state changes with force, the width of the probability function should change with the loading rate [47].

As mentioned above, dynamic force spectra often reveal a nonlinear dependence with the logarithm of the loading rate. This was initially interpreted by Evans as the crossing of multiple barriers along the dissociation pathway, each one described by a logarithmic dependence with different slope [54]. More recently, the development of the so-called microscopic theories suggested that nonlinear spectra may reflect the actual shape of the energy landscape and some models have been proposed $[47,48,55]$. In addition, some works proposed the existence of different regimes depending on the pulling rate. At pulling rates faster than the internal relaxation of the molecular bond, mainly accessible in molecular dynamics (MD) simulations and perhaps high-speed force spectroscopy, a square root dependence has been predicted [49, 56]. The interpretation of this fast dynamic regime, sometimes referred as deterministic, is still under debate. At the other dynamic end, at low pulling rates and if rebinding is not negligible, near-equilibrium regimes have been proposed where rupture forces are independent of the applied loading rate [50].

\subsection{Investigation of Single-Molecule Mechanics via The Computational Microscope}

The existing experimental methodologies for probing single molecules provide limited microscopic details. Atomistic level understanding of single-molecule pulling experiments is possible using molecular simulations. Molecular dynamics (MD) simulations provide an atomistic description of a classical many-body system. The term classical defines the motion of the particles within the system investigated as subject to the laws of classical mechanics. In MD simulations, numerical methods are used to solve Newton's equation of motion for a series 
of finite time steps. It uses an empirically derived potential energy function describing all molecular interactions [57]. The generated atomistic coordinates of the system, the so called trajectory, can be considered as deterministic and reproducible given the exact initial conditions and time. However, the time resolution of most of the biomolecular processes is significantly higher than the accessible timescales via conventional fully atomistic MD simulations within tolerable time of computation. Several sophisticated methods have been developed to overcome this size and time limitation of classical MD simulations such as coarse graining the description of the system, replica exchange molecular dynamics, parallel tempering, metadynamics, umbrella sampling, steered MD, simulated annealing, etc. [58].

\subsubsection{Molecular Dynamics Simulation}

The methodology of Molecular Dynamics Simulations is based on classical mechanics and consists of solving Newton's equation of motion for a system of $N$ interacting particles:

$$
F_{i}=-\nabla_{r_{i}} V=m_{i} a_{i}, \quad i=1,2, \ldots, N
$$

where $V$ is the potential energy, $F_{i}$ is the force exerted on the particle $i, m_{i}$ is its mass and $a_{i}$ is its acceleration. Knowledge of the force on each atom allows determination of the acceleration of each atom in the system. Integration of the second term of the equation yields a trajectory that describes the positions, velocities and accelerations of the particles as they vary with time. The method is deterministic; meaning that knowing the positions and velocities of each atom, prediction of the state of the system at any time; future or past, is possible. Using this methodology, successive configurations of the system can be generated. In intermolecular interactions, the force applied to each particle changes whenever the particle itself or any other particle in interaction with it changes its position. This feature is implemented to the simulation with the use of a continuous potential, in which the motions of all particles are coupled [59]. At 
this stage, since molecular systems generally consist of a vast number of particles, it becomes impossible to find the properties of such a complex system analytically. Therefore, MD simulation integrates the equations by using numerical methods. The most common algorithm used in integration is Verlet algorithm [60]. Positions and accelerations at a time $t$ and positions from time $(t-\delta t)$ are used to calculate new positions at time $(t+\delta t)$. Here, $\delta t$ is called time step and is typically 2 femtoseconds in all atomistic MD simulations. Smaller time steps increase the accuracy of this numerical integration with the cost of increased computational time and power whereas using too high time steps causes instabilities in the simulations. Then the velocities can be obtained from the difference in positions. From generated values, new positions can be obtained successively. The leapfrog algorithm [61], which is a slightly modified version of Verlet method, velocity Verlet method [62] and the Beeman algorithm [63] are also popular integrators used in MD simulations.

The force field defines the potential energy of a system $V$ by a mathematical formula with the associated parameters in the context of MD simulations, which gives $a_{i}$ through Equation 1.5, as a function of the atomic positions/coordinates. $F_{i}$ in Equation 1.5 are obtained from derivatives of the potential function [57,64-67]. The potential energy of particle $i$ is defined in terms of interactions between bonded atoms, bond angle and torsional angle potentials, and electrostatic and van der Waals interactions between non-bonded atoms as:

$$
\begin{gathered}
V=\sum_{\text {bonds }} \frac{k_{i}^{\text {bond }}}{2}\left(r_{i}-r_{0}\right)^{2}+\sum_{\text {angles }} \frac{k_{i}^{\text {angle }}}{2}\left(\theta_{i}-\theta_{0}\right)^{2}+ \\
\sum_{\text {torsions }} \frac{k_{i}^{\text {tors }}}{2}\left(1+\cos \left(n_{i} \phi_{i}-\delta_{i}\right)\right)+
\end{gathered}
$$




$$
\sum_{i}^{N} \sum_{j \neq i}^{N}\left(4 \epsilon_{i j}\left[\left(\frac{\sigma_{i j}}{r_{i j}}\right)^{12}-\left(\frac{\sigma_{i j}}{r_{i j}}\right)^{6}\right]+\frac{q_{i} q_{j}}{4 \pi \epsilon_{0} r_{i j}}\right)
$$

The first term in the equation describes the interaction of pairs of bonded atoms. The second term is similarly the summation over all the angles in the molecule modelled using a harmonic potential, where $\theta_{i}$ is the angle between the three successive atoms. The torsional potential describes the change in energy when a bond rotates, and is described by the third term. The fourth contribution is for the non-bonded atoms, which are separated by at least three atoms. The non-bonded interactions are defined by two different potentials. The former one is the Lennard-Jones 12-6 potential function that accounts for van der Waals interactions, whereas the latter one is the Coulomb potential for electrostatic interactions [68].

There are a number of different force fields developed for different purposes and implemented in various MD simulation software. AMBER [69], CHARMM [66], GROMOS [70], OPLS [71] and COMPASS [72] are the most common force fields used in molecular dynamics simulations. Whereas the first three are mostly used for simulating biomolecules, the last two are for condensed matter simulations. Since their first development, all the force fields are continuously evolving with improvements and there are several stable versions [73].

As can be seen from the structure of the algorithm, to start an MD simulation and generate configurations of the system, the initial state should be defined. Either experimental or theoretical inputs can be used at this stage. In addition to the positions of the elements of the network, the initial velocities should also be defined. Experimental inputs are generally used for the atomistic coordinates of a structure, in the form of X-ray or NMR structures. The structure is subjected to energy minimization before starting the simulation. The initial velocities are generated by theoretical methods. A commonly used method is randomly 
selecting the initial velocities from a Maxwell-Boltzmann distribution, for the specified temperature [59].

There are a considerable number of MD software packages available to use today. AMBER [74], CHARMM [75], GROMACS [76, 77] and NAMD [78] are the most commonly used packages. Among these, NAMD has a performance advantage because of its success in parallel computing. NAMD is also distributed free of charge. In addition, NAMD is well integrated with VMD [79], a molecular visualization software package. This facilitates the analysis of MD output files and even allows the user to perform interactive MD simulation [80].

\subsubsection{Steered Molecular Dynamics (SMD)}

The Steered Molecular Dynamics (SMD) method was developed to mimic AFM pulling experiments, where external forces are applied to probe biomolecules in addition to their intrinsic forces defined in classical MD simulations [81, 82]. SMD simulations have been used extensively to explore the mechanics of biomolecular processes such as unbinding and unfolding at single-molecule level [67, 81, 83-88]

SMD has two common protocols: constant force and constant velocity pulling. In constant force SMD, a constant force is directly applied to a specific atom or a group of atoms, referred to as the SMD atom, in addition to the force field potential. In constant velocity SMD, a harmonic potential (a virtual spring) is attached between a specific atom or group of atoms and a fictitious point (dummy atom) and this dummy atom is moved at constant velocity in a chosen direction to provide motion along the reaction coordinate. The force induced by this harmonic spring becomes

$$
\vec{F}_{S M D}(\vec{r})=-\nabla V_{S M D}(\vec{r})
$$


where the harmonic potential added to the force field potential is

$$
V_{S M D}(\vec{r})=\frac{1}{2} k\left[v t-\left(\vec{r}-\vec{r}_{0}\right) \vec{n}\right]^{2}
$$

where $k$ is the spring constant, $v$ is the pulling velocity, $\vec{r}$ is the actual position of SMD atom, $\vec{r}_{0}$ is the initial position of SMD atom, and $\vec{n}$ is the pulling direction [81]. In both protocols, all atoms in the system adjust to the forced change in the structure and/or conformations along a particular pathway.

Selection of pulling direction or a series of directions is one of the crucial parameters in SMD simulations. Although a straight-line path is sufficient in most of the simulations such as unfolding, the SMD simulations of ligand unbinding from the receptor requires change in the pulling direction along the simulation to avoid distortion of the receptor [89]. Different strategies for selecting the pulling direction are applied in the literature [90-92]. Allowing partial unfolding along the unbinding simulations of molecules, which is physically relevant in some molecular interactions, is still challenging in the field of molecular simulations.

As the simulation progresses, force and extension as a function of time data will be collected. Different quantitative measurements can be calculated from the simulation trajectory, such as elastic properties, potential of mean force along the pulling direction, reaction kinetics parameters, the free energy difference between two states via Jarzynski's equality [93, 94], and, of course, rupture forces [95]. The calculated potential of mean force (PMF) is the interpretation of the free energy change along the chosen reaction coordinates for the progress. SMD simulation is a non-equilibrium process and therefore the equilibrium descriptions such as calculation of PMF cannot be applied directly, although several approaches have been proposed 
originated from Jarzynski's equality, such as the Hummer-Szabo approach [96] and successfully applied in the simulations [97-99].

The limitations in computational resources and the limitations in the technology used in AFM instruments lead to a discrepancy in time scale between computational and experimental investigations. The pulling velocities used in SMD simulations are about 6 orders of magnitude faster than the velocities used in conventional AFM pulling experiments. Thus, the forces calculated from SMD simulations are higher than those obtained from AFM experiments [95]. However, this does not mean that SMD simulations provide incorrect values $[67,100]$. With the development of high-speed force spectroscopy (HS-FS), the gap between experiments and simulations tends to vanish and a good agreement between simulations and experiments has been observed for protein unfolding [12]. From the computational point of view, using coarse grained (CG) models may provide simulations at experimental speeds with reasonable computational time and resources. There are successful applications of CG models by Gō-like potentials to study mechanical properties of proteins near experimental pulling velocities [101, 102]. Multiscale modelling of the simulation system combined with enhanced sampling simulations such as SMD is still a promising approach to simulate biomolecular processes under applied forces at experimental and physiological velocities. The continuous development in AFM technology and MD algorithms together with increasing computational power will allow a perfect match between experimental and simulated DFS data in the near future. Hopefully, by combining both approaches more refined theories will become available.

\section{Materials}

\subsection{Equipment}

1. Silicon nitride cantilevers.

2. Glass surface (cover slips). 
3. Ozone or plasma cleaner for cleaning cantilevers

4. Oven to bake cantilevers and glass surfaces

5. Fine stainless steel tweezers for handling cantilevers

6. Pyrex Petri dishes/similar inert vessel for treating cantilevers with acetone, acids, and other reactive reagents.

7. 24-well tissue culture plate for rinsing and treating cantilevers

8. Parafilm

9. Plastic petri dishes

10. AFM

\subsection{Chemicals}

1. Nanopure MilliQ water.

2. Phosphate Buffered Saline (PBS): $10 \mathrm{mM} \mathrm{Na}_{2} \mathrm{HPO}_{4}, 1.76 \mathrm{mM} \mathrm{KH}_{2} \mathrm{PO}_{4}, 137 \mathrm{mM}$ $\mathrm{NaCl}, 2.7 \mathrm{mM} \mathrm{KCl} \mathrm{pH:9} \mathrm{and} \mathrm{pH}: 7.2$

3. HPLC grade or $>95 \%$ purity acetone.

4. Analytical grade or $>99.9 \%$ purity ethanol (EtOH).

5. Piranha solution ( $75 \%$ sulfuric acid $\left(\mathrm{H}_{2} \mathrm{SO}_{4}\right)$ and $25 \%$ hydrogen peroxide $\left(\mathrm{H}_{2} \mathrm{O}_{2}\right)$

6. $\mathrm{O}_{2}$, Argon and $\mathrm{N}_{2}$ gas

\section{Methods}

\subsection{Surface functionalization to probe streptavidin-biotin complexes}

Here, we describe a protocol to functionalize silicon or silicon nitride cantilevers with biotin. Biotin is covalently attached to the cantilever through a polyethylene glycol (PEG) linker, which provides a total length $\sim 10 \mathrm{~nm}$. We use a silane-PEG-biotin molecule (1 kDa, Nanocs 
Inc, NY) that minimizes the number of steps required for covalent attachment to the AFM tip. The protocol is simple.

1. Rinse the glass surfaces and cantilevers with acetone for 10 minutes in a clean pyrex/glass petri dish under a well-ventilated hood. (see Notes 4-6)

2. If necessary (in case they are used) immerse the glass surfaces in a mixture of sulfuric acid $\left(\mathrm{H}_{2} \mathrm{SO}_{4}\right)(75 \%)$ and hydrogen peroxide $\left(\mathrm{H}_{2} \mathrm{O}_{2}\right)(25 \%)$ (so-called piranha solution) for 30 minutes. (see Notes 7-9)

3. Rinse the glass surfaces by dipping into $\sim 1 \mathrm{ml}$ milli-Q water in a 24 -well tissue culture plate (5 times)

4. Dry the glass surfaces and cantilevers with a gentle flow of $\mathrm{N}_{2}$

5. Clean glass surfaces and silicon nitride cantilevers with plasma cleaner $80 \mathrm{~W}$ power under oxygen for 5 minutes at 0.6 mbar. This is a crucial step, $\mathrm{O}_{2}$ plasma oxidizes the silicon surface allowing the attachment of silane groups in the next step. The color of $\mathrm{O}_{2}$ plasma should be white indicating a high level of $\mathrm{O}_{2}$.

6. Immerse cantilevers in a solution of $10-20 \mathrm{mg} / \mathrm{ml}$ silane-PEG-biotin in ethanol/water $(95 / 5)$.

7. Incubate for 2 hours.

8. Rinse cantilevers with ethanol and ultrapure water and stored at $4^{\circ} \mathrm{C}$ until use.

In the case of streptavidin/biotin, several products are commercially available with both biotin or streptavidin crosslinked to their surface, such as polystyrene beads, agarose beads (also called resins in the context of protein purification) and flat surfaces. The material of the surface will determine the amount of unspecific binding [103]. Here, we use streptavidin polystyrene beads. To immobilize streptavidin beads on the surface, we first coat the surface using 
biotinylated bovine serum albumin $0.5 \mathrm{mg} / \mathrm{ml}$ in $100 \mathrm{mM} \mathrm{NaHCO}, \mathrm{pH} 8.6$ overnight in a $4^{\circ} \mathrm{C}$ humidified chamber, and then deposit the beads and let adhere. After this step, rinsing has to be carried out with caution to avoid bead detachment.

PEG molecules have a characteristic force response upon stretching and allow easy identification by their stretching length $[14,27]$ (Fig. 3). Thus, knowing the length of the linker provides a fingerprint to discern between specific and non-specific interactions (Fig. 3a).

\subsection{Calibration of AFM cantilevers}

The procedure for spring constant and invOLS calibration using the non-contact method is as follows:

1. Mount the cantilever on the cantilever holder.

2. Focus the laser beam at the very end of the cantilever where the tip is positioned trying to maximize the sum signal on the photodiode.

3. Adjust the position of the reflected beam to the center of the bi-segmented photodiode (zero horizontal deflection).

4. Acquire and save the thermal fluctuation spectrum of the cantilever. (see Note 10)

5. Extract the resonance frequency and the quality factor from the power spectral density (PSD) of the thermal fluctuation response of a cantilever (See Figure 2.b).

6. Calculate the spring constant of the cantilever using Sader formula by using measured width and length of the cantilever (using optical or electron microscopy). (see Notes 11-12)

7. After functionalization of the cantilever, prior to engaging the cantilever on the 
surface, repeat Steps 2-5 and record and save the thermal fluctuation spectrum in liquid.

8. Using the calculated spring constant at Step 6 and the PSD in liquid (See Figure 2.c), determine the invOLS value.

\subsection{Force Spectroscopy Measurements}

After calibration and functionalization of the AFM cantilever, force spectroscopy measurements are acquired by using the detailed procedure described below. We propose the use of precalibrated spring constants to apply the contact-free method of sensitivity calibration.

1. Place the sample (coated surface) on the AFM stage with $100 \mu 1$ of measurement buffer (PBS) to form a drop.

2. Mount the cantilever on the cantilever holder taking care to not let it dry, as this may damage the linked biomolecule.

3. Immediately immerse the cantilever in the measurement buffer on the protein-coated surface to minimize any contact with air.

4. Dip the cantilever into the buffer drop to form a liquid meniscus, ensuring that the tip is sufficiently away from the surface (at least $20 \mu \mathrm{m}$ )

5. Focus the laser beam at the end of the cantilever trying to maximize the sum signal on the photodiode.

6. Adjust the position of the reflected beam near the center of the segmented photodiode (zero vertical and horizontal deflection).

7. Acquire the thermal fluctuation spectrum of the cantilever in liquid away from the surface to calibrate the optical lever sensitivity using the known spring constant. (see Note 10)

8. Determine the invOLS using the known spring constant. 
9. Engage the cantilever on the surface trying to minimize the applied force and time in contact.

10. Acquire force-distance curves with the following steps

a. Approach the tip to the surface at $\sim 1 \mu \mathrm{m} / \mathrm{s}$ (constant velocity) with feedback control until the required force is reached $(\sim 100 \mathrm{pN})$.

b. Maintain this force for the desired contact time.

c. Vary the contact time to reach an adhesion frequency (fraction of successful binding events) of $\sim 10 \%$. Low adhesion frequency ensures that most of the unbinding events are due to single receptor/ligand complexes. (see Note 13)

d. Retract to initial position with constant velocity.

e. Move within the XY plane between force curves to probe fresh regions and prevent surface degradation.

f. Repeat steps a to e.

g. Continue collecting the data until the statistically significant amount of unbinding force-distance curves is reached (50-100 successful events per velocity).

h. Repeat the force-distance data collection at different logarithmically spaced velocities, covering the widest range of velocities possible (usually from $\sim 10 \mathrm{~nm} / \mathrm{s}$ to $\sim 10 \mu \mathrm{m} / \mathrm{s}$, but can go up to $\mathrm{mm} / \mathrm{s}$ using HS-AFM).

\subsection{Data Processing and Analysis}

Thousands of force-distance curves are collected during DFS experiments. The unbinding forces are determined by inspecting the individual force-extension curves. However, the large number of data collected during the experiments and the low probability of acquisition of meaningful single-molecule unbinding events requires an automated or semi-automated data processing tool. Engineering the biomolecules or linkers so they provide a well-defined 
fingerprint with known unfolding or extension profile helps automated data processing. Polyethylene glycol (PEG) is often used in this regard. There have been successful developments and implementations that allow automated analysis of DFS data, reducing the bias introduced by manual approaches [104-108]. Most of the commercially available AFM manufacturers now provide now their own software which is capable of processing the forcedistance curves in a semi-automated fashion, but we recommend writing one's own tool, as it provides high flexibility and control on all the applied data processing [29]. For that, it is necessary to pool the unbinding events according to loading rate and determine the most probable rupture forces. The details of DFS data analysis are described below.

1. Divide the whole range of loading rates from all rupture events into equal intervals in logarithmic space to get uniform spacing between points.

2. Pool rupture force data according to the loading rate intervals in step 11.

3. Plot the rupture forces as histograms for each loading rate interval (Fig. 3b).

4. Fit a Gaussian distribution to the force histograms to get the most probable rupture force (MPRF) per loading rate interval. (see Note 22)

5. Fit a lognormal distribution function to the loading rates histogram for each interval to obtain the most probable loading rate or use the median value.

6. Plot the most probable rupture forces versus loading rate to obtain the dynamic force spectrum of the interaction (Fig. 3c).

7. Apply a theoretical model describing the dependence of rupture force and loading rate. (see Note 23)

8. Determine the kinetic parameters dissociation rate at zero force $\left(k_{o f f}\right)$ and the distance to the transition state $\left(x_{\beta}\right)$ of the unbinding energy landscape.

\subsection{SMD Simulation}


A detailed procedure for constant velocity SMD simulation using NAMD is as follows:

1. Download the necessary open source software packages to your computer. (see Note 24-25)

2. Download or generate the required files to run the simulation as follows:

a. Obtain your Protein Data Bank (pdb) file containing the atomic coordinates of your system. (see Note 26)

b. Construct your Protein Structure File (psf) in VMD using appropriate topology parameters that contains all the structural parameters of your protein. Before creating your psf file, determine the protonation state of each histidine residue in your system and correct their names corresponding to their protonation states.

c. Solvate and ionize your simulation system according to your choice of simulation box using VMD.

d. Download the appropriate force field parameter file defining the bonds strengths, equilibrium distances etc. that will be used in your simulation. NAMD supports CHARMM, AMBER, XPLOR, and GROMOS force fields.

e. Generate your configuration file containing all the options that NAMD will adopt to run the simulation.

3. Perform an energy minimization to non-physiological crystal packing forces and to obtain the minimum energy structure.

4. Perform conventional MD simulation until the system is equilibrated (unbiased equilibration) and extract the equilibrated state of your system. If the potential of mean force (PMF) calculation is desired for constant velocity pulling simulations, then a biased equilibration should also be performed.

5. Create a reference file containing information about the fixed and SMD atom(s) by duplicating the equilibrated pdb file. (see Note 27) 
6. Modify the NAMD configuration file by adding necessary parameters for the SMD simulation. (see Note 28)

7. Run your simulation.

8. Analyse the trajectory by extracting the acquired quantitative parameters such as rupture force and loading rate, that can be compared to experimental data.

\section{Notes}

1. Negative or positive deflections are, of course, relative.

2. Some commercial AFM software packages are now applying the Sader method on the thermal spectrum in liquid, at the same time used to determine the invOLS. Although interesting because it may avoid one step, the validity of the Sader method for low Qfactor peaks should be first addressed.

3. Notice that, while the Bell-Evans model is defined for the most probable value of the rupture force, other models describe the mean value.

4. Glass cover slips are recommended for better functionalization.

5. Since cantilevers are quite small it is a difficult task to handle them and it is common to lose some of them during the whole coating process. Thus, we recommend functionalizing more than one cantilever for each experiment. In addition, it is recommended to check whether the cantilevers on the chip are intact and undamaged before starting the functionalization protocol. If you coat more than one probe simultaneously, label the probes before calibration. A tungsten or diamond pen may be used for that.

6. When working with acetone or ethanol, pyrex/glass petri dishes and pipets should be used to prevent corrosion and should be performed under well-ventilated hood. 
7. The mixing process of $\mathrm{H}_{2} \mathrm{O}_{2}$ and $\mathrm{H}_{2} \mathrm{SO}_{4}$ is a highly exothermic reaction. In order to avoid any boiling or splashing, $\mathrm{H}_{2} \mathrm{O}_{2}$ must be mixed with $\mathrm{H}_{2} \mathrm{SO}_{4}$, SLOWLY. The solution itself is highly explosive and hazardous, therefore the necessary safety precautions must be followed. Piranha solution cannot be stored for future use.

8. Since the piranha solution is highly corrosive, only glass or Teflon tools must be used while working with piranha solution.

9. The cantilevers should not be cleaned with piranha solution because they easily flip and break and it may damage the gold coating.

10. The cantilever must be sufficiently away from any surface, at least $50 \mu \mathrm{m}$.

11. Sader method is valid only for high $Q$-factor cantilevers.

12. Sader and co-workers initiated a web-based platform for spring constant calibration, called the Global Calibration Initiative (GCI). Using this portal, any AFM user can upload the calibration parameters (spring constant, resonance frequency and Q-factor) of their own cantilevers, establishing a global database. The assessment of the uploaded data from individual users facilitates calculation of a universal coefficient, called $A$ coefficient, for each specific cantilever geometry which completes the functional relation between the spring constant $(k)$, the resonance frequency $\left(f_{R}\right)$ and the quality factor $(Q)$ measured in air. The portal also allows correction of the spring constant using the globally calculated $A$-coefficient. The spring constant determination via the GCI becomes more and more accurate as users upload their own calibrations to the database.

13. The adhesion frequency (fraction of successful unfolding events) has to be kept below $30 \%$, preferable $10 \%$ in general. Moreover, the contact time between adhering surfaces and/or the protein densities on the surfaces can be adjusted to control the adhesion frequency. 
14. After rupture, the signal should be a straight, flat line with noise caused by thermal fluctuations until the end of the piezo movement. A signal presenting a slope is often observed on tip moving AFM systems and long z-ranges. Fitting a straight line to this part of the curve will give the baseline.

15. The actual tip displacement is different than the piezo movement because of the bending of the cantilever due to the viscous forces acting on the cantilever caused my surrounding fluid movement.

16. The intersection of the smoothed retract trace and the extrapolation of the corrected baseline is a way to determine the point of contact. Another method is to use the first data point that changes from positive to negative deflection (starting from the contact part).

17. Note that this velocity is the velocity of the tip relative to the liquid.

18. Due to the viscous effects of the surrounding medium and depending on the retraction velocity, the distance to the surface and the cantilever geometry, the baselines of the approach and retract traces can be shifted to each other. The difference between these two traces is due to the viscous drag. If this difference is important, the viscous drag effect should be corrected. The difference in force between approach and retraction baselines will give the viscous drag force. The viscous drag coefficient $(b)$ is calculated by dividing the viscous drag force by the total velocity (approach plus retraction velocities). Due to wall effects, this coefficient increases near the flat surface with a distance $(h)$, the dependence of which varies according to cantilever geometry and tip height $[109,110]$.

19. The derivative is very sensitive to noise, thus only apply this correction if the viscous effect is important (>10\% of the unbinding forces). Smoothing of the velocity signal might be required. 
20. Sharp jumps after a stretching regime in the force-distance profile are candidates for rupture events of protein-ligand complexes. The first derivative of the deflection provides the slope of the curve, large slope values reflect sharp jumps. Defining a threshold for the slope is commonly used to locate inflection points and thereby the location of the peaks.

21. If the slope in the retraction trace is in line with the slope of the retract prior to the contact point, then the peaks can be considered as due to non-specific adhesion (Fig. 3a, bottom trace). As mentioned before, using linker molecules like PEG can help to differentiate the peaks arising from specific interactions from the non-specific ones by comparing the distance before the rupture and the shape of the stretching regime (Fig. 3a, three top traces). Fitting a polymer elasticity model such as the worm-like chain (WLC) $[111,112]$, the freely jointed chain (FJC) [113], or the freely rotating chain (FRC) [114] model, which allows determination of mechanical stretching profile and thereby the contour length of the extension, can also be used in this step.

22. Depending on the behavior of the molecular interaction of protein and ligand a bimodal distribution may be present. In that case, the first peak is likely due to single ruptures.

23. The first and still the most common phenomenological model, used to describe the disruption of receptor-ligand bonds by an applied mechanical force is the Bell model [115], which was further developed for non-constant loading forces by Evans and Ritchie (Bell-Evans model) [116]. The model describes a linear dependence between the rupture force and the logarithm of the loading rate. The Bell model has been extended further to include the modulation of the distance between initial state and transient state [47], the spring constant of the cantilever and linker system [117, 118] and even the possibility of rebinding [119], and to extend the applicable dynamic range 
$[56,120]$, predicting nonlinear relationships between the most probable rupture force and the logarithm of the loading rate.

24. NAMD in order to run molecular dynamics simulations. Select the appropriate version based on your platform at http://www.ks.uiuc.edu/Research/namd/.

25. VMD in order to prepare the system to simulate, visualize and analyse the simulation generated trajectory. Select the appropriate version based on your platform at http://www.ks.uiuc.edu/Research/vmd/

26. You can either download the most suitable and readily available pdb files from http://www.pdb.org or construct your own pdb file by hand in the same format.

27. Set the occupancy column of the reference file to 1 for SMD atom(s) and the rest to 0 . Then set the B column of the pdb file to 1 for fixed atom(s) and the rest to 0 . Other columns can also be used for defining the fixed atom(s) as is specified in the configuration file.

28. For instance, enable the Fixed Atom Constraint and SMD block that contains the pulling velocity, the spring constant of the virtual spring between the dummy atom and SMD atom and the pulling direction and disable the temperature control.

\section{References}

1. $\quad$ Egan, P., et al., The role of mechanics in biological and bio-inspired systems. Nature Communications, 2015. 6(May): p. 7418-7418.

2. Boehr, D.D. and R. Nussinov, The role of dynamic conformational ensembles in biomolecular recognition. Nature chemical biology, 2009. 5(11): p. 789-796.

3. Kamm, R., J. Lammerding, and M. Mofrad, Cellular Nanomechanics. 2010. p. 11711200.

4. Zhang, X., et al., Atomic Force Microscopy of Protein-Protein Interactions. 2009, Springer US: New York, NY. p. 555-570.

5. Wennmalm, S. and S.M. Simon, Studying individual events in biology. Annual review of biochemistry, 2007. 76: p. 419-446. 
6. Greenleaf, W.J., M.T. Woodside, and S.M. Block, High-resolution, single-molecule measurements of biomolecular motion. Annual review of biophysics and biomolecular structure, 2007. 36: p. 171-90.

7. Binnig, G., C. Quate, and C. Gerber, Atomic Force Microscope, 1986. p. 930-933.

8. Barkai, E., Y. Jung, and R. Silbey, Theory of single-molecule spectroscopy: beyond the ensemble average. Annual review of physical chemistry, 2004. 55(1): p. 457-507.

9. Bustamante, C., J.C. Macosko, and G.J. Wuite, Grabbing the cat by the tail: manipulating molecules one by one. Nature reviews. Molecular cell biology, 2000. 1(2): p. $130-136$.

10. Neuman, K.C. and A. Nagy, Single-molecule force spectroscopy: optical tweezers, magnetic tweezers and atomic force microscopy. Nature methods, 2008. 5(6): p. 491505.

11. Eghiaian, F., et al., High-speed atomic force microscopy: imaging and force spectroscopy. FEBS letters, 2014. 588(19): p. 3631-8.

12. Rico, F., et al., High-Speed Force Spectroscopy Molecular Dynamics Simulations. Science, 2013. 342: p. 741-743.

13. Yu, H., et al., Hidden dynamics in the unfolding of individual bacteriorhodopsin proteins. Science, 2017. 355(6328): p. 945-950.

14. Oesterhelt, F., et al., Unfolding Pathways of Individual Bacteriorhodopsins. Science, 2000. 288(5463): p. 143-146.

15. Florin, E.L., V.T. Moy, and H.E. Gaub, Adhesion forces between individual ligandreceptor pairs. Science (New York, N.Y.), 1994. 264(5157): p. 415-417.

16. Moy, V.T., E.L. Florin, and H.E. Gaub, Intermolecular forces and energies between ligands and receptors. Science, 1994. 266(5183): p. 257-259.

17. Rief, M., et al., Reversible unfolding of individual titin immunoglobulin domains by AFM. Science, 1997. 276(5315): p. 1109-12.

18. Rief, M., et al., Single Molecule Force Spectroscopy on Polysaccharides by Atomic Force Microscopy. Science, 1997. 275(5304): p. 1295-7.

19. Lee, G.U., D.a. Kidwell, and R.J. Colton, Sensing Discrete Streptavidin-Biotin Interactions with Atomic Force Microscopy. Langmuir, 1994. 2(9): p. 354-357.

20. Lee, G.U., L.A. Chrisey, and R.J. Colton, DIRECT MEASUREMENT OF THE FORCES BETWEEN COMPLEMENTARY STRANDS OF DNA. Science, 1994. 266(5186): p. 771-773.

21. Lekka, M., et al., Elasticity of normal and cancerous human bladder cells studied by scanning force microscopy. European biophysics journal : EBJ, 1999. 28(4): p. 312316. 
22. Wu, H.W., T. Kuhn, and V.T. Moy, Mechanical properties of 1929 cells measured by atomic force microscopy: Effects of anticytoskeletal drugs and membrane crosslinking. Scanning, 1998. 20(5): p. 389-397.

23. Zhang, X., E. Wojcikiewicz, and V.T. Moy, Force spectroscopy of the leukocyte function-associated antigen-1/intercellular adhesion molecule-1 interaction. Biophysical journal, 2002. 83(4): p. 2270-2279.

24. Benoit, M., et al., Discrete interactions in cell adhesion measured by single-molecule force spectroscopy. Nature Cell Biology, 2000. 2(6): p. 313-317.

25. Rico, F., Molecular to cellular mechanics. Insights from atomic force micrsocpy, 2016, Aix-Marseille Université.

26. Hinterdorfer, P. and Y.F. Dufrene, Detection and localization of single molecular recognition events using atomic force microscopy. Nat Methods, 2006. 3(5): p. 347-55.

27. Hinterdorfer, P., et al., Detection and localization of individual antibody-antigen recognition events by atomic force microscopy. Proc Natl Acad Sci U S A, 1996. 93(8): p. 3477-81.

28. Sulchek, T.A., et al., Dynamic force spectroscopy of parallel individual Mucin1antibody bonds. Proceedings of the National Academy of Sciences of the United States of America, 2005. 102(46): p. 16638-16643.

29. Ott, W., et al., Single-molecule force spectroscopy on polyproteins and receptor-ligand complexes: The current toolbox. Journal of Structural Biology, 2015.

30. Putman, C.A.J., et al., A Detailed Analysis of the Optical Beam Deflection Technique for Use in Atomic Force Microscopy. Journal of Applied Physics, 1992. 72(1): p. 6-12.

31. Proksch, R., et al., Finite optical spot size and position corrections in thermal spring constant calibration. Nanotechnology, 2004. 15(9): p. 1344-1350.

32. Hansma, P.K., et al., A new, optical-lever based atomic force microscope. Journal of Applied Physics, 1994. 76(2): p. 796-799.

33. Ohler, B., Cantilever spring constant calibration using laser Doppler vibrometry. The Review of scientific instruments, 2007. 78(6): p. 063701-063701.

34. Ohler, B., Practical Advice on the Determination of Cantilever Spring Constants. Spring, 2007. AN94: p. 1-12.

35. Hutter, J.L. and J. Bechhoefer, Calibration of atomic-force microscope tips. Review of Scientific Instruments, 1993. 64(7): p. 1868-1873.

36. Butt, H.J. and M. Jaschke, Calculation of thermal noise in atomic force microscopy. Nanotechnology, 1995. 6: p. 1-7.

37. Pirzer, T. and T. Hugel, Atomic force microscopy spring constant determination in viscous liquids. Review of Scientific Instruments, 2009. 80(3): p. 035110-6. 
38. Higgins, M.J., et al., Noninvasive determination of optical lever sensitivity in atomic force microscopy. Review of Scientific Instruments, 2006. 77(1): p. 1-5.

39. Stark, R.W., T. Drobek, and W.M. Heckl, Thermomechanical noise of a free v-shaped cantilever for atomic-force microscopy. Ultramicroscopy, 2001. 86(1-2): p. 207-215.

40. Sader, J.E., J.W.M. Chon, and P. Mulvaney, Calibration of rectangular atomic force microscope cantilevers. Review of Scientific Instruments, 1999. 70(10): p. 3967-3969.

41. Sader, J.E., et al., Spring constant calibration of atomic force microscope cantilevers of arbitrary shape. Review of Scientific Instruments, 2012. 83(10): p. 103705.

42. Sader, J.E., J. Lu, and P. Mulvaney, Effect of cantilever geometry on the optical lever sensitivities and thermal noise method of the atomic force microscope. Review of Scientific Instruments, 2014. 85(11): p. 6-11.

43. Sader, J.E., et al., A virtual instrument to standardise the calibration of atomic force microscope cantilevers. Review of Scientific Instruments, 2016. 87(9): p. 093711.

44. Heim, L.O., M. Kappl, and H.J. Butt, Tilt of atomic force microscope cantilevers: Effect on spring constant and adhesion measurements. Langmuir, 2004. 20(7): p. 2760-2764.

45. Hutter, J.L., Comment on tilt of atomic force microscope cantilevers: effect on spring constant and adhesion measurements. Langmuir, 2005. 21(6): p. 2630-2.

46. Rigato, A., et al., High-frequency microrheology reveals cytoskeleton dynamics in living cells. Nature Physics, 2017.

47. Dudko, O.K., G. Hummer, and A. Szabo, Intrinsic rates and activation free energies from single-molecule pulling experiments. Physical Review Letters, 2006. 96(10).

48. Dudko, O.K., G. Hummer, and A. Szabo, Theory, analysis, and interpretation of singlemolecule force spectroscopy experiments. Proc Natl Acad Sci U S A, 2008. 105(41): p. 15755-60.

49. Sturm, S., J.T. Bullerjahn, and K. Kroy, Intramolecular relaxation in dynamic force spectroscopy. The European Physical Journal Special Topics, 2014. 223(14): p. 31293144.

50. Friddle, R.W., Theoretical Models in Force Spectroscopy. Dynamic Force Spectroscopy and Biomolecular Recognition, 2012.

51. Noy, A. and R.W. Friddle, Practical single molecule force spectroscopy: How to determine fundamental thermodynamic parameters of intermolecular bonds with an atomic force microscope. Methods, 2013. 60(2): p. 142-150.

52. Kramers, H.A., Brownian motion in a field of force and the diffusion model of chemical reactions. Physica, 1940. 7(4): p. 284-304.

53. Hanggi, P., P. Talkner, and M. Borkovec, Reaction-Rate Theory - 50 Years after Kramers. Reviews of Modern Physics, 1990. 62(2): p. 251-341. 
54. Merkel, R., et al., Energy landscapes of receptor-ligand bonds explored with dynamic force spectroscopy. Nature, 1999. 397(January): p. 50-53.

55. Friddle, R.W., Unified model of dynamic forced barrier crossing in single molecules. Physical Review Letters, 2008. 100(13).

56. Hummer, G. and A. Szabo, Kinetics from nonequilibrium single-molecule pulling experiments. Biophys J, 2003. 85(1): p. 5-15.

57. McCammon, J.A., B.R. Gelin, and M. Karplus, Dynamics of folded proteins. Nature, 1977. 267(5612): p. 585-590.

58. Pfaendtner, J. and M. Bonomi, Efficient Sampling of High-Dimensional Free-Energy Landscapes with Parallel Bias Metadynamics. Journal of Chemical Theory and Computation, 2015: p. 151009124113008-151009124113008.

59. Leach, A.R., Molecular Modelling: Principles and Applications. 2nd ed. 2001: Prentice Hall.

60. Verlet, L., Computer "experiments" on classical fluids. I. Thermodynamical properties of Lennard-Jones molecules. Physical Review, 1967. 159(1): p. 98-103.

61. Hockney, R.W., The potential calculation and some applications(Potential calculation from given source distribution, including direct and iterative methods, error analysis. Meth. Compt. Phys, 1970. 9: p. 136-211.

62. Swope, W.C., et al., A computer simulation method for the calculation of equilibrium constants for the formation of physical clusters of molecules: Application to small water clusters. J. Chem. Phys., 1982. 76(1): p. 637-649.

63. Beeman, D., Some multistep methods for use in molecular dynamics calculations. Journal of Computational Physics, 1976. 20(2): p. 130-139.

64. Karplus, M. and G.a. Petsko, Molecular dynamics simulations in biology. Nature, 1990. 347(6294): p. 631-639.

65. Daggett, V. and M. Levitt, Realistic simulations of native-protein dynamics in solution and beyond. Annual review of biophysics and biomolecular structure, 1993. 22: p. 35380 .

66. Mackerell, a.D., et al., All-Atom Empirical Potential for Molecular Modeling and Dynamics Studies of Proteins. J Phys Chem B, 1998. 102(97): p. 3586-3616.

67. Sotomayor, M. and K. Schulten, Single-molecule experiments in vitro and in silico. Science (New York, N.Y.), 2007. 316(5828): p. 1144-1148.

68. Adcock, S.a. and J.A. McCammon, Molecular Dynamics : Survey of Methods for Simulating the Activity of Proteins. Chemical Reviews, 2006. 106(February): p. 15891615.

69. Cornell, W.D., et al., A Second Generation Force Field for the Simulation of Proteins, Nucleic Acids, and Organic Molecules. J Am Chem Soc, 1996. 118(9): p. 2309-2309. 
70. Oostenbrink, C., et al., A biomolecular force field based on the free enthalpy of hydration and solvation: The GROMOS force-field parameter sets $53 A 5$ and 53A6. Journal of Computational Chemistry, 2004. 25(13): p. 1656-1676.

71. Jorgensen, W.L., D.S. Maxwell, and J. Tirado-Rives, Development and testing of the OPLS all-atom force field on conformational energetics and properties of organic liquids. Journal of the American Chemical Society, 1996. 118(45): p. 11225-11236.

72. Sun, H., COMPASS: An ab Initio Force-Field Optimized for Condensed-Phase ApplicationssOverview with Details on Alkane and Benzene Compounds. Journal of Physical Chemistry B, 1998. 5647(98): p. 7338-7364.

73. González, M.A., Force fields and molecular dynamics simulations. Collection SFN, 2011. 12: p. 169-200.

74. Pearlman, D.A., et al., AMBER, a computer program for applying molecular mechanics, normal mode analysis, molecular dynamics and free energy calculations to elucidate the structures and energies of molecules. Comp. Phys. Comm., 1995. 91: p. 1-41.

75. Brooks, B.R., et al., CHARMM: A program for macromolecular energy, minimization, and dynamics calculations. Journal of Computational Chemistry, 1983. 4(2): p. 187217.

76. Berendsen, H.J.C., D. van der Spoel, and R. van Drunen, GROMACS: A messagepassing parallel molecular dynamics implementation. Computer Physics Communications, 1995. 91(1-3): p. 43-56.

77. Lindahl, E., B. Hess, and D. van der Spoel, GROMACS 3.0: a package for molecular simulation and trajectory analysis. J Mol Model, 2001. 7: p. 306-317.

78. Phillips, J.C., et al., Scalable molecular dynamics with NAMD. Journal of Computational Chemistry, 2005. 26(16): p. 1781-1802.

79. Humphrey, W., A. Dalke, and K. Schulten, VMD: Visual molecular dynamics. Journal of Molecular Graphics, 1996. 14(1): p. 33-38.

80. Ribeiro, J.V., et al., QwikMD - Integrative Molecular Dynamics Toolkit for Novices and Experts. Scientific Reports, 2016. 6(May): p. 26536-26536.

81. Isralewitz, B., M. Gao, and K. Schulten, Steered molecular dynamics and mechanical functions of proteins. Current Opinion in Structural Biology, 2001. 11(2): p. 224-230.

82. Grubmüller, H., Force probe molecular dynamics simulations. Methods in molecular biology (Clifton, N.J.), 2005. 305(1): p. 493-515.

83. Grater, F., et al., Mechanically induced titin kinase activation studied by force-probe molecular dynamics simulations. Biophys J, 2005. 88(2): p. 790-804.

84. Rief, M. and H. Grubmuller, Force spectroscopy of single biomolecules. Chemphyschem, 2002. 3(3): p. 255-61. 
85. Grubmuller, H., B. Heymann, and P. Tavan, Ligand binding: Molecular mechanics calculation of the streptavidin biotin rupture force. Science, 1996. 271(5251): p. 997999.

86. Lee, E.H., et al., Discovery Through the Computational Microscope. Structure, 2009. 17(10): p. 1295-1306.

87. Lu, H. and K. Schulten, The Key Event in Force-Induced Unfolding of Titin's Immunoglobulin Domains. Biophysical Journal, 2000. 79(1): p. 51-65.

88. Izrailev, S., et al., Molecular dynamics study of unbinding of the avidin-biotin complex. Biophysical Journal, 1997. 72(4): p. 1568-1581.

89. Franca, E.F., A.M. Amarante, and F.L. Leite, Introduction to Atomic Force Microscopy Simulation. Microscopy: Science, Technology, Applications and Education, 2010: p. 1338-1349.

90. Ludemann, S.K., V. Lounnas, and R.C. Wade, How do substrates enter and products exit the buried active site of cytochrome P450cam? 1. Random expulsion molecular dynamics investigation of ligand access channels and mechanisms. Journal of Molecular Biology, 2000. 303(5): p. 797-811.

91. Kosztin, D., S. Izrailev, and K. Schulten, Unbinding of retinoic acid from its receptor studied by steered molecular dynamics. Biophysical journal, 1999. 76(1 Pt 1): p. 18897.

92. Izrailev, S., et al., Steered Molecular Dynamics. Computational Molecular Dynamics: Challenges, Methods, Ideas SE - 2, 1999. 4: p. 39-65.

93. Jarzynski, C., Nonequilibrium Equality for Free Energy Differences. Physical Review Letters, 1997. 78(14): p. 2690-2693.

94. Jarzynski, C., Equilibrium free-energy differences from nonequilibrium measurements: A master-equation approach. Physical Review E, 1997. 56(5): p. 5018-5035.

95. Gao, M., et al., Molecular mechanisms of cellular mechanics. Physical Chemistry Chemical Physics, 2006. 8(32): p. 3692-3706.

96. Hummer, G. and A. Szabo, Free energy reconstruction from nonequilibrium singlemolecule pulling experiments. Proc Natl Acad Sci U S A, 2001. 98(7): p. 3658-61.

97. Balsera, M., et al., Reconstructing potential energy functions from simulated forceinduced unbinding processes. Biophysical journal, 1997. 73(3): p. 1281-7.

98. Gullingsrud, J., R. Braun, and K. Schulten, Reconstructing potentials of mean force through time series analysis of steered molecular dynamics simulations. J. Comput. Phys., 1999. 151: p. 190-211.

99. Park, S., et al., Free energy calculation from steered molecular dynamics simulations using Jarzynski's equality. The Journal of Chemical Physics, 2003. 119(6): p. 35593566. 
100. Sotomayor, M., Computational exploration of single-protein mechanics by steered molecular dynamics. 2015. 030001: p. 030001-030001.

101. Zhmurov, A., et al., SOP-GPU: Accelerating biomolecular simulations in the centisecond timescale using graphics processors. Proteins: Structure, Function and Bioinformatics, 2010. 78(14): p. 2984-2999.

102. Kmiecik, S., et al., Coarse-Grained Modeling of Protein Dynamics. Computational Methods to Study the Structure and Dynamics of Biomolecules, 2014. 1: p. 55-79.

103. Celik, E. and V.T. Moy, Nonspecific interactions in AFM force spectroscopy measurements. Journal of Molecular Recognition, 2012. 25(1): p. 53-56.

104. Kuhn, M., et al., Automated alignment and pattern recognition of single-molecule force spectroscopy data. Journal of Microscopy, 2005. 218(2): p. 125-132.

105. Puchner, E.M., et al., Comparing proteins by their unfolding pattern. Biophys J, 2008. 109(50): p. 426-434.

106. Fuhrmann, A., et al., Refined procedure of evaluating experimental single-molecule force spectroscopy data. Physical Review E - Statistical, Nonlinear, and Soft Matter Physics, 2008. 77(3): p. 1-10.

107. Bosshart, P.D., P.L.T.M. Frederix, and A. Engel, Reference-free alignment and sorting of single-molecule force spectroscopy data. Biophysical Journal, 2012. 102(9): p. 22022211.

108. Jobst, M.A., et al., Resolving dual binding conformations of cellulosome cohesindockerin complexes using single-molecule force spectroscopy. eLife, 2015. 4(e10319).

109. Alcaraz, J., et al., Correction of microrheological measurements of soft samples with atomic force microscopy for the hydrodynamic drag on the cantilever. Langmuir, 2002. 18(3): p. 716-721.

110. Janovjak, H.J., J. Struckmeier, and D.J. Muller, Hydrodynamic effects in fast AFM single-molecule force measurements. European Biophysics Journal with Biophysics Letters, 2005. 34(1): p. 91-96.

111. Rief, M., et al., Reversible Unfolding of Individual Titin Immunoglobulin Domains by AFM. Science, 1997. 276: p. 1109-1112.

112. Bustamante, C., et al., Entropic elasticity of lambda-phage DNA. Science (New York, N.Y.), 1994. 265(1994): p. 1599-1600.

113. Ortiz, C. and G. Hadziioannou, Entropic elasticity of single polymer chains of poly(methacrylic acid) measured by atomic force microscopy. Macromol., 1999. 32: p. 780-787.

114. Livadaru, L., R.R. Netz, and H.J. Kreuzer, Stretching response of discrete semiflexible polymers. Macromolecules, 2003. 36(10): p. 3732-3744. 
115. Bell, G., Models for the specific adhesion of cells to cells. Science, 1978. 200(4342): p. 618-627.

116. Evans, E. and K. Ritchie, Dynamic Strength of Molecular Adhesion Bonds. Biophysical Journal, 1997. 72: p. 1541-1555.

117. Evans, E. and K. Ritchie, Strength of a weak bond connecting flexible polymer chains. Biophys J, 1999. 76(5): p. 2439-47.

118. Maitra, A. and G. Arya, Model accounting for the effects of pulling-device stiffness in the analyses of single-molecule force measurements. Phys Rev Lett, 2010. 104(10): p. 108301.

119. Friddle, R.W., a. Noy, and J.J. De Yoreo, Interpreting the widespread nonlinear force spectra of intermolecular bonds. Proceedings of the National Academy of Sciences, 2012. 109(34): p. 13573-13578.

120. Bullerjahn, J.T., S. Sturm, and K. Kroy, Theory of rapid force spectroscopy. Nat Commun, 2014. 5. 


\section{Figures}

a Atomic Force Microscopy (AFM)

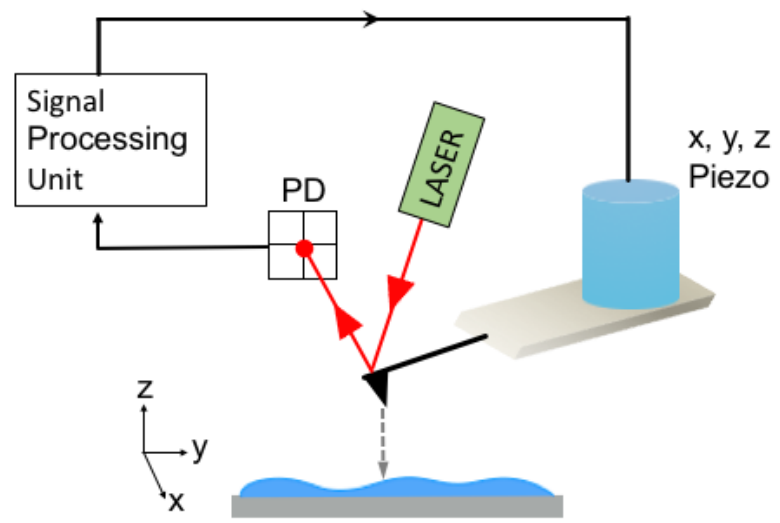

b

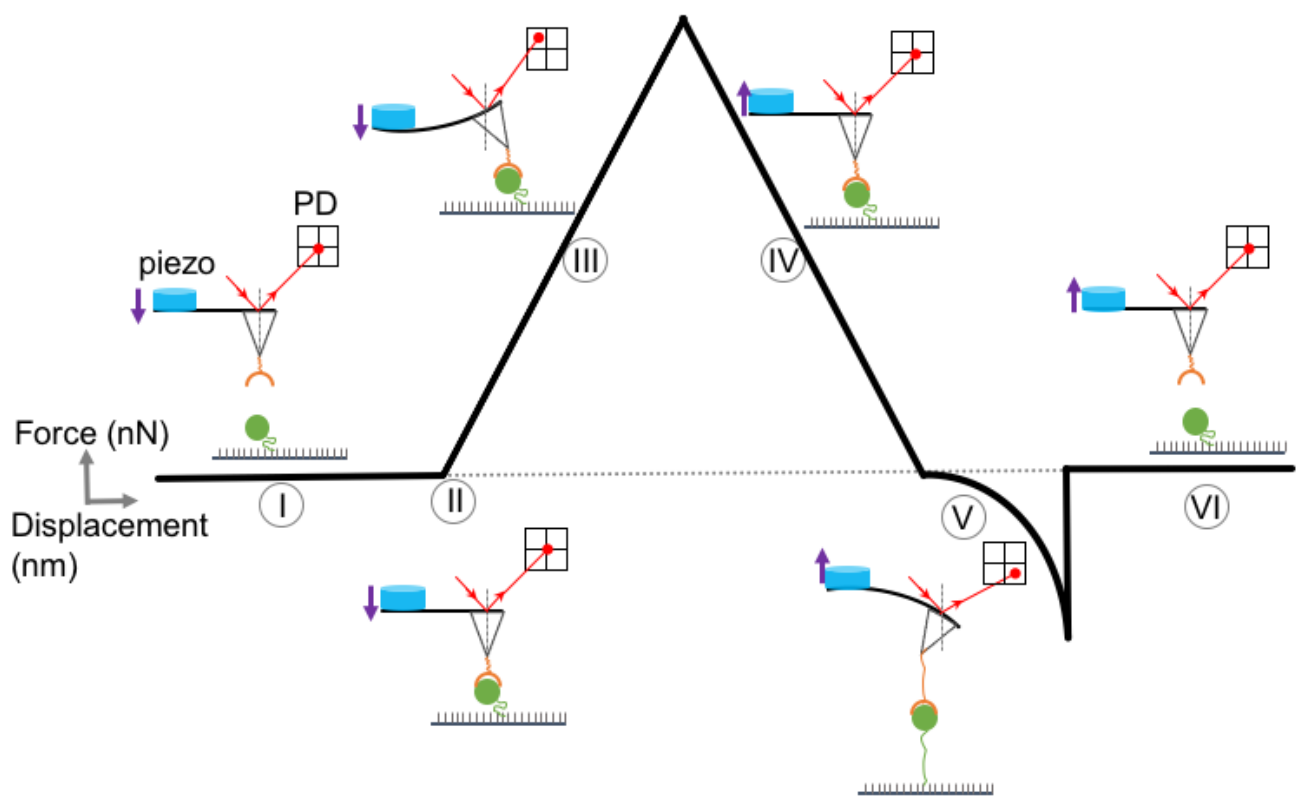

Figure 1. A schematic of the AFM and force measurement a. Schematic illustration of the fundamental components of an AFM device with working principles. b. A schematic photodetector signal of the cantilever deflection recorded during a biomolecular force spectroscopy curve together with the corresponding position of cantilever and surface. 
a

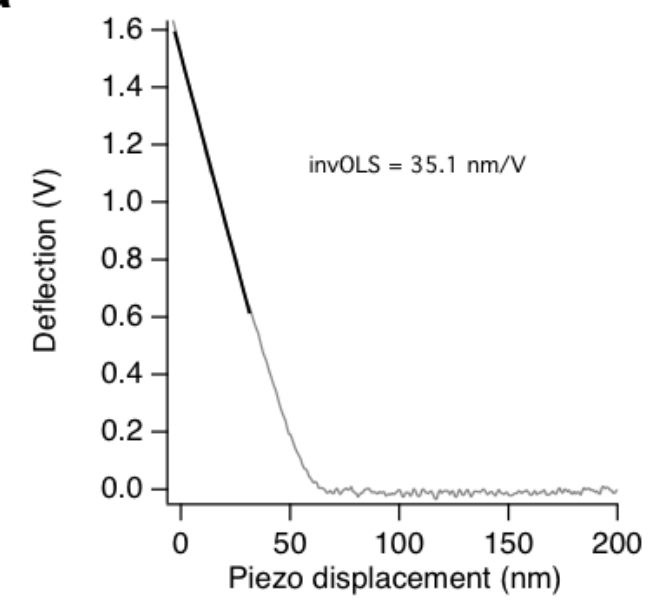

b

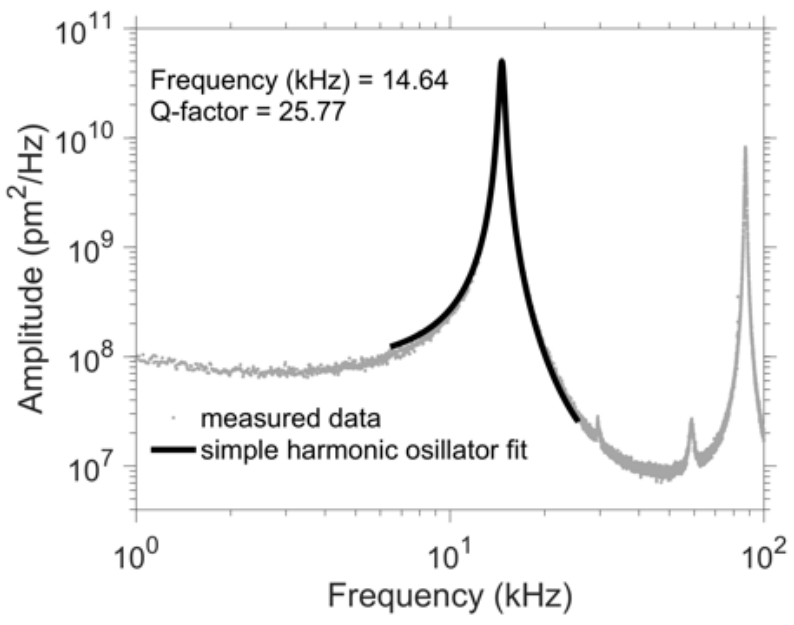

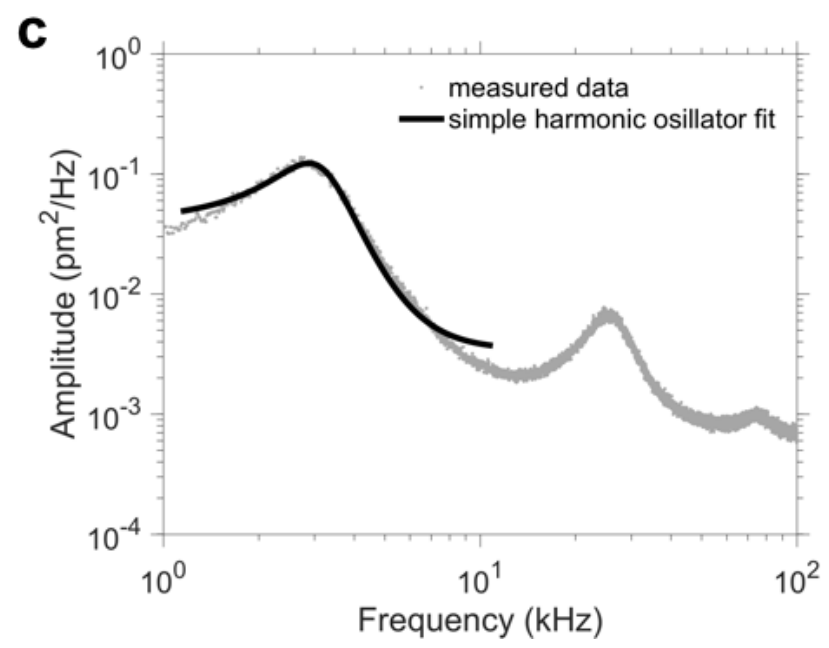

Figure 2. Calibration of AFM cantilevers. a. Calibration of sensitivity. Representative curve obtained on a hard substrate. The slope calculated from the linear part of the curve was used to obtain the optical lever sensitivity, where OLS=1/invOLS. b. Power spectral density (PSD) of the thermal fluctuations of a cantilever (Bruker MLCT-D) in air with the respective fit to cantilever's first mode. The spring constant is determined using the Sader method and the fitted resonance frequency and Q-factor obtained from this result. c. PSD of the thermal fluctuations of a cantilever (Bruker MLCT-D) in liquid with the respective fit to cantilever's first mode. The PSD in liquid can be used to extract the spring constant knowing the invOLS value or, vice versa. 

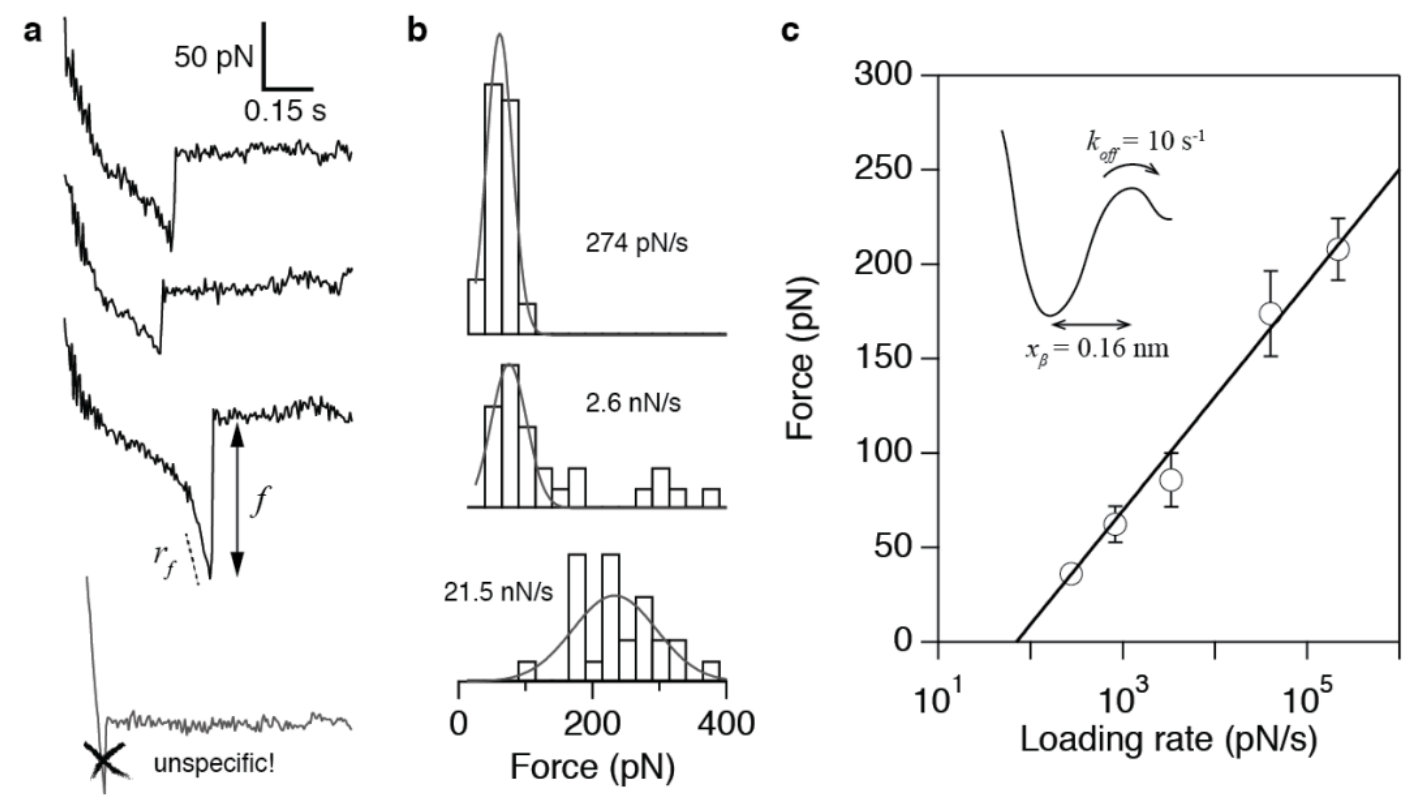

Figure 3. Dynamic force spectroscopy data. a. Representative force-time curves (top) showing specific unbinding after the stretching of the PEG linker. The rupture force is the determined relative to the zero force level $(f)$ and the loading rate from the slope just before rupture $(r f)$. The bottom curve shows an example of a force-time curve likely due to unspecific binding of the tip with the substrate (notice that no stretching regime is observed and the slope is the same during contact and pulling regimes). b. Histograms of rupture forces at three different loading rates with the corresponding Gaussian curve to extract the most probable rupture force. c. Dynamic force spectrum showing the most probable rupture forces as a function of the loading rate (open symbols). The solid line represents the fit of the Bell-Evans model Eq. 3.4 to extract the parameters of the energy landscape (inset): distance to the transition state $\left(x_{\beta}\right)$ and intrinsic dissociation rate at zero force $\left(k_{\mathrm{off}}\right)$. Error bars represent the standard error of the mean. 